\title{
Analgesia Pós-Operatória em Pacientes Pediátricos: Estudo Comparativo entre Anestésico Local, Opióides e Antiinflamatório não Esteróide *
}

\section{Postoperative Analgesia in Pediatric Patients: Comparative Study among Local Anesthetics, Opioids and Non-Steroidal Anti-Inflammatory Drugs}

\author{
Miriam Seligman Menezes, TSA ${ }^{1}$, Judymara Lauzi Gozzani, TSA ${ }^{2}$
}

\section{RESUMO}

Menezes MS, Gozzani JL - Analgesia Pós-Operatória em Pacientes Pediátricos: Estudo Comparativo entre Anestésico Local, Opióides e Antiinflamatório não Esteróide

Justificativa e Objetivos - O tratamento da dor pós-operatória em crianças tem merecido atenção especial nas últimas décadas. O propósito deste estudo foi analisar a analgesia pós-operatória de crianças no que se relaciona à qualidade e à duração da analgesia, à confiabilidade dos métodos de avaliação e à incidência de efeitos colaterais decorrentes das diferentes técnicas de analgesia utilizadas.

Método - Participaram do estudo 100 crianças com idades entre 2 e 12 anos alocadas em 5 grupos de 20 crianças cada, que receberam, logo após a indução da anestesia, os seguintes tratamentos de analgesia: grupo B, bupivacaína a $0,25 \%$, com vasoconstritor, 0,5 a $1 \mathrm{ml} . \mathrm{kg}^{-1}$; grupo $\mathrm{F}$, fentanil, $1,5 \mu \mathrm{g} \cdot \mathrm{kg}^{-1}$; grupo $M$, morfina, $30 \mu \mathrm{g} \cdot \mathrm{kg}^{-1}$, grupo S, sufentanil, $0,3 \mu \mathrm{g} \cdot \mathrm{kg}^{-1}$, todos por via peridural caudal e o grupo $D$, que recebeu diclofenaco potássico (1 $\left.\mathrm{mg} \mathrm{kg}^{-1}\right)$ por via retal. A dor foi avaliada por 2 métodos distintos: um predominantemente comportamental, objetivo e o outro de auto-avaliação, subjetivo, durante as primeiras 4 horas e a partir deste momento até a $24^{a}$ hora. Efeitos colaterais foram observados e tratados.

Resultados - Nas primeiras 4 horas os pacientes dos grupos $B$, $F, M$ e $S$ apresentaram comportamentos semelhantes, com mínimas necessidades de analgesia complementar. Nas 20 horas restantes o maior tempo de analgesia foi o observado no grupo $S$, não diferindo dos grupos $F$ e $M$, mas sendo significativamente superior ao tempo dos grupos $B$ e $D$. Diclofenaco retal não promoveu alívio efetivo da dor. Maior incidência de efeitos colaterais ocorreu no grupo $M$ que não diferiu do grupo $S$, mas foi significativamente superior aos grupos $F, B$ e D. Houve correlação positiva e significativa entre os escores das 2 escalas de avaliação de dor.

* Recebido do (Received from) Hospital São Paulo da Universidade Federal do Estado de São Paulo (UNIFESP) e Hospital Universitário de Santa Maria da Universidade Federal de Santa Maria (UFSM), RS

1. Professora Adjunta da Disciplina de Anestesiologia da UFSM; Doutorado em Anestesiologia pela UNIFESP; Responsável pelo CET Prof. Manoel Alvarez - UFSM

2. Mestrado em Biologia Molecular pela UNIFESP; Doutorado em Anestesiologia pela UNIFESP

Apresentado (Submitted) em 11 de junho de 2001

Aceito (Accepted) para publicação em 28 de agosto de 2001

Correspondência para (Mail to)

Dra. Miriam Seligman Menezes

Rua Estácio de Lemos, 190

97010-150 Santa Maria, RS

(C) Sociedade Brasileira de Anestesiologia, 2002
Conclusões - Os opióides espinhais mostraram-se seguros e efetivos na analgesia pós-operatória em crianças, porém quando comparados à bupivacaína não apresentaram diferenças relevantes e apresentaram maior incidência de efeitos colaterais. O diclofenaco por via retal não se mostrou efetivo como analgésico único quando comparado às outras técnicas.

UNITERMOS - ANALGÉSICOS, Opióides: fentanil, morfina, sufentanil, Antiinflamatórios: diclofenaco; ANESTESIA, Pediátrica; ANESTÉSICOS, Local: bupivacaína; DOR, Aguda: pós-operatória; TÉCNICAS ANESTÉSICAS, Regional: peridural caudal

\section{SUMMARY}

Menezes MS, Gozzani JL - Postoperative Analgesia in Pediatric Patients: Comparative Study among Local Anesthetics, Opioids and Non-Steroidal Anti-Inflammatory Drugs

Background and Objectives - The treatment of postoperative pain in children has been given special attention in the last decades. This study aimed at analyzing postoperative analgesia in children, considering analgesia quality and duration, evaluation methods reliability and the incidence of side effects resulting from different analgesia techniques.

Methods - Participated in this study 100 children, aged 2 to 12 years, allocated in 5 groups of 20 children each, who received, right after anesthesia, the following analgesics: group $B$, $0.25 \%$ bupivacaine with epinephrine $(1: 400,000) 0.5$ to 1 $\mathrm{ml} . \mathrm{kg}^{-1}$; group F, $1.5 \mu \mathrm{g} . \mathrm{kg}^{-1}$ fentanyl; group $\mathrm{M}, 30 \mu \mathrm{g} . \mathrm{kg}^{-1}$ morphine; group S, $0.3 \mu \mathrm{g} \cdot \mathrm{kg}^{-1}$ sufentanil, all by epidural caudal block and group $D$, who received rectal diclofenac $\left(1 \mathrm{mg} \cdot \mathrm{kg}^{-1}\right)$. Pain was evaluated by two different methods: one, predominantly behavioral and objective and the other subjective and self-evaluated, during the first 4 hours and then up to the $24^{\text {th }}$ hour. Side effects were observed and treated.

Results - In the first 4 hours, groups B, F, M and S patients presented similar behavior, with a minimum need for complementary analgesia. In the remaining 20 hours, the longest analgesia duration was seen in group $S$, which did not differ from groups $F$ and $M$, but was significantly longer than for groups $B$ and $D$. Rectal diclofenac has not promoted effective pain relief. There has been a higher incidence of side-effects in group $M$, which did not differ from group $S$, but was significantly higher than for groups $F, B$ and $D$. There has been a positive and significant correlation between both pain scales.

Conclusions - Spinal opioids are safe and effective for postoperative analgesia in children, but when compared to bupivacaine, there have been no significant differences and a higher incidence of side effects. Rectal diclofenac was not effective as a single analgesic as compared to other techniques.

KEY WORDS - ANALGESICS, Opioids: fentanyl, morphine, sufentanil, Anti-inflammatory: diclofenac; ANESTHESIA, Pediatric; ANESTHETICS, Local: bupivacaine; ANESTHETIC TECHNIQUES, Regional: caudal epidural; PAIN, Acute: postoperative 


\section{INTRODUÇÃO}

A partir da constatação de que crianças apresentam percepção, resposta e memorização da dor de maneira semelhante aos adultos, diferentes técnicas de analgesia para alívio da dor, especialmente da dor pós-operatória, têm sido propostas com o objetivo de proteger as crianças das alterações metabólicas, hemodinâmicas e psicológicas decorrentes dos procedimentos cirúrgicos ${ }^{1-5}$.

A avaliação da dor vem sendo apontada como uma das principais dificuldades no manuseio da dor pós-operatória, em crianças com idade inferior a 6 anos, o que tem levado os anestesiologistas a escolherem técnicas que promovam analgesia antes mesmo do despertar dos pacientes, facilitando, de certa maneira, esta avaliação ${ }^{6-8}$.

A administração de analgésicos sistêmicos, por diferentes vias e a utilização de bloqueios locorregionais têm sido as técnicas usuais para a abordagem terapêutica da dor pós-operatória em crianças, embora deva ser considerado, ainda hoje, o temor das equipes médicas e de enfermagem ao uso de opióides em crianças, pelos riscos de efeitos colaterais.

O objetivo deste trabalho foi avaliar e comparar a analgesia pós-operatória em crianças, obtida com diferentes técnicas de analgesia. Estudou-se a bupivacaína, a morfina, o fentanil e o sufentanil, todos por via peridural caudal e o diclofenaco potássico, por via retal, administrados depois da indução da anestesia, observando-se a qualidade da analgesia nas primeiras 4 horas através de 2 métodos distintos de avaliação da intensidade dolorosa, a confiabilidade dos métodos de avaliação utilizados, a duração total da analgesia e a incidência de efeitos colaterais, nas primeiras 24 horas de pós-operatório.

\section{MÉTODO}

Após a aprovação pela Comissão de Ética e o consentimento escrito dos pais, 100 crianças, com idades entre 2 e 12 anos, estado físico ASAI, II e III, de ambos os sexos, programadas para procedimentos cirúrgicos ortopédicos de membros inferiores, urogenitais, anais e abdominais infraumbilicais, foram incluídas no estudo, de modo sistemático, ou seja, os 2 primeiros pertenceriam ao Grupo B, os 2 seguintes ao Grupo F e assim sucessivamente, passando pelos Grupos M, S e D até perfazerem um total de 20 pacientes em cada grupo.

Medicação pré-anestésica, quando necessária, constou de midazolam $\left(0,5 \mathrm{mg} \cdot \mathrm{kg}^{-1}\right)$, por via oral ou nasal, 20 ou $30 \mathrm{minu}$ tos antes do procedimento cirúrgico. A indução da anestesia foi realizada ou com halotano, em concentrações crescentes e óxido nitroso e oxigênio (1:1), ou com tiopental por via venosa, e a manutenção foi realizada com os mesmos agentes inalatórios, através de tubo orotraqueal, máscara laríngea ou cânula orofaríngea, com ventilação assistida ou controla$\mathrm{da}$, em sistema sem reabsorvedor de $\mathrm{CO}_{2}$. Succinilcolina, atracúrio ou plano profundo de anestesia inalatória foram os meios utilizados para facilitar a intubação traqueal. Amonitorização constou de cardioscopia, oximetria de pulso, este- toscópio pré-cordial e medidas não invasivas de pressão arterial.

Logo após a indução da anestesia, nos pacientes do grupo $B$ foi realizado bloqueio peridural caudal com bupivacaína a

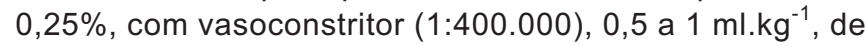
acordo com o local da incisão cirúrgica; no grupo $F$, foi realizado bloqueio peridural caudal com fentanil, $\left(1,5 \mu \mathrm{g} \cdot \mathrm{kg}^{-1}\right)$, diluído em $10 \mathrm{ml}$ de solução fisiológica; pacientes do grupo $\mathrm{M}$, receberam morfina $\left(30 \mu \mathrm{g} . \mathrm{kg}^{-1}\right)$, num volume de $10 \mathrm{ml}$ de solução fisiológica, por via peridural caudal; pela mesma via, pacientes do grupo $S$ receberam sufentanil $\left(0,3 \mu \mathrm{g} \cdot \mathrm{kg}^{-1}\right)$, diluído em volume de $10 \mathrm{ml}$ de solução fisiológica. Nos pacientes do grupo $D$, foi administrado supositório de diclofenaco potássico, por via retal, na dose de $1 \mathrm{mg} \cdot \mathrm{kg}^{-1}$.

No período pós-operatório imediato, todos os pacientes receberam oxigênio através de máscara facial e foram monitorizados com oxímetro de pulso. Durante as primeiras 4 horas de pós-operatório foram utilizadas 2 escalas distintas para avaliação da dor: Escala de Avaliação de Dor e Desconforto $(\text { EADD })^{9}$ apresentada na tabela I e Escala de Auto-avaliação $(E A A)^{10}$, conforme figura 1 , sendo as avaliações efetuadas a cada 15 minutos na primeira hora e, após este período, a cada 60 minutos até a quarta hora, e administrados analgésicos por via oral sempre que as crianças alcançassem escores iguais ou superiores a 7 na Escala de Avaliação de Dor e Desconforto. A partir deste período, a avaliação era feita pela enfermagem e/ou pais, com base na observação de mudança de comportamento e/ou postura das crianças, tentando relacionar estes dados com a presença de dor e necessidade de complementação com analgésicos. Acetaminofen (10 a $\left.15 \mathrm{mg} \cdot \mathrm{kg}^{-1}\right)$ por via oral, metoclopramida $\left(0,15 \mathrm{mg} \cdot \mathrm{kg}^{-1}\right)$ e/ou naloxona $\left(0,5\right.$ a $\left.4 \mu \mathrm{g} \cdot \mathrm{kg}^{-1}\right)$, ambos por via venosa, foram preconizados para utilização em casos de dor, náusea e/ou vômito e outros efeitos colaterais advindos do uso de opióides.

Tabela I - Escala de Avaliação de Dor e Desconforto (EADD)

\begin{tabular}{lll}
\hline Parâmetros & Critérios & Pontos \\
\hline Pressão arterial & Até $20 \%$ dos valores pré-operatórios & 0 \\
& $20-30 \%$ dos valores pré-operatórios & 1 \\
& $>30 \%$ dos valores pré-operatórios & 2 \\
Choro & Sem choro & 0 \\
& Choro, respondendo a carinhos & 1 \\
Movimentos & Choro, não respondendo a carinhos & 2 \\
& Nenhum & 0 \\
Ansiedade & Inquieto & 1 \\
& Debatendo-se & 2 \\
& Dormindo ou calmo & 0 \\
& Moderada & 1 \\
& Muito ansioso & 2 \\
& Sem postura específica & 0 \\
& Flexão de pernas e coxas & 1 \\
& Segurando região operada & 2 \\
& Não referindo dor & 0 \\
& Dor não localizada & 1 \\
& Dor localizada & 2 \\
\hline
\end{tabular}

Revista Brasileira de Anestesiologia Vol. 52, № 2, Março - Abril, 2002 


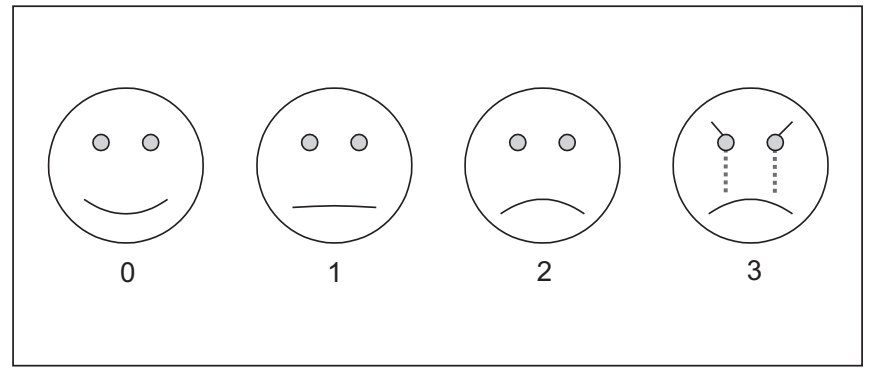

Figura 1 - Escala de Auto-Avaliação (EAA)

A análise estatística foi realizada utilizando teste $\mathrm{Fe} \mathrm{X}^{2}$ para comparar as variações demográficas entre os grupos; teste de Kruskal-Wallis para comparação de médias de escores de dor e de tempo de analgesia entre os grupos; teste de $\mathrm{X}^{2}$ para avaliar a necessidade de complementação de analgésicos nas primeiras 4 horas e a incidência de efeitos colaterais em cada grupo e o estudo de correlação de Pearson para avaliar a correlação entre as escalas de avaliação de dor uti- lizadas. O valor de $p<0,05$ foi considerado como mínimo estatisticamente significativo.

\section{RESULTADOS}

Não houve diferenças demográficas significativas entre os pacientes dos 5 grupos estudados (Tabela II).

Ocorreram diferenças significativas nas médias de escores de dor, entre os grupos, em ambas as escalas, até os $60 \mathrm{mi}-$ nutos de avaliação; após este período, os escores de dor não diferiram significativamente. Escores de dor dos pacientes do Grupo $D$ foram superiores aos escores de dor dos pacientes dos demais grupos aos 30, 45 e 60 minutos iniciais de avaliação (Tabela III).

Pacientes dos grupos B, F, M e S apresentaram comportamento semelhante em relação à reduzida necessidade de analgesia suplementar, nas primeiras horas de avaliação, diferindo significativamente $(p<0,05)$ dos pacientes do grupo

Tabela II - Dados Demográficos

\begin{tabular}{|c|c|c|c|c|c|}
\hline & Grupo B & Grupo F & Grupo M & Grupo S & Grupo D \\
\hline Idade (meses) * & $\begin{array}{c}67,80 \pm 29,04 \\
(25-120)\end{array}$ & $\begin{array}{c}68,45 \pm 40,60 \\
(24-136)\end{array}$ & $\begin{array}{c}65,45 \pm 31,69 \\
(27-133)\end{array}$ & $\begin{array}{c}80,15 \pm 22,07 \\
48-127)\end{array}$ & $\begin{array}{c}73,40 \pm 36,19 \\
(28-144)\end{array}$ \\
\hline Peso $(\mathrm{kg})^{*}$ & $\begin{array}{c}19,67 \pm 6,26 \\
(11-30)\end{array}$ & $\begin{array}{c}21,22 \pm 10,29 \\
(10-44)\end{array}$ & $\begin{array}{c}18,06 \pm 6,05 \\
(9,5-30)\end{array}$ & $\begin{array}{c}22,92 \pm 7,33 \\
(10,3-41)\end{array}$ & $\begin{array}{c}20,10 \pm 7,33 \\
(12-44)\end{array}$ \\
\hline \multicolumn{6}{|l|}{ Sexo } \\
\hline Masculino & 15 & 18 & 14 & 15 & 15 \\
\hline Feminino & 5 & 2 & 6 & 5 & 5 \\
\hline \multicolumn{6}{|l|}{ ASA } \\
\hline I & 16 & 17 & 16 & 16 & 18 \\
\hline II & 2 & 3 & 2 & 3 & 2 \\
\hline III & 2 & - & 2 & 1 & - \\
\hline
\end{tabular}

*Valores expressos pela Média \pm DP e valores mínimos e máximos entre parênteses; $p<0,05$ pelos testes $F$ e Qui-quadrado

Tabela III - Comparação entre os Grupos por Escores de Dor, pelos Métodos das Escalas de Avaliação de Dor e Desconforto (EADD) e de Auto-avaliação (EAA)

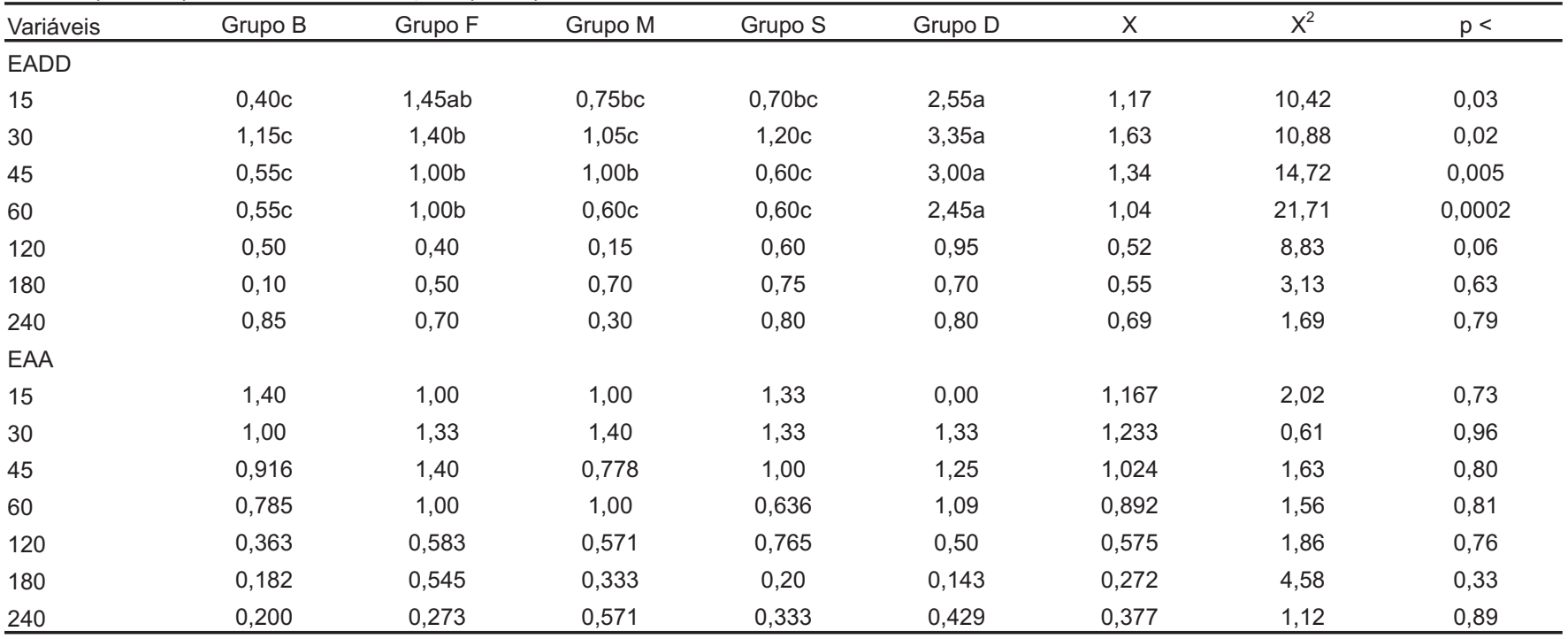

Médias seguidas de letras diferentes são significativamente diferentes, pelo teste de Kruskal-Wallis $(p<0,05)$ 
$D$, em que a necessidade de complementação com analgésicos, nas primeiras 4 horas, foi muito elevada (Figura 2). O tempo total de analgesia nas 24 horas foi reduzido no Grupo $D$, com diferença estatisticamente significativa em relação aos demais grupos (Figura 3). Maior tempo de analgesia foi observado nos pacientes do grupo S, não diferindo significativamente dos grupos $\mathrm{Fe} \mathrm{M}$, mas sim dos pacientes dos grupos D e B. Não foram verificadas diferenças significativas no tempo de analgesia entre os pacientes dos grupos M, F e B.

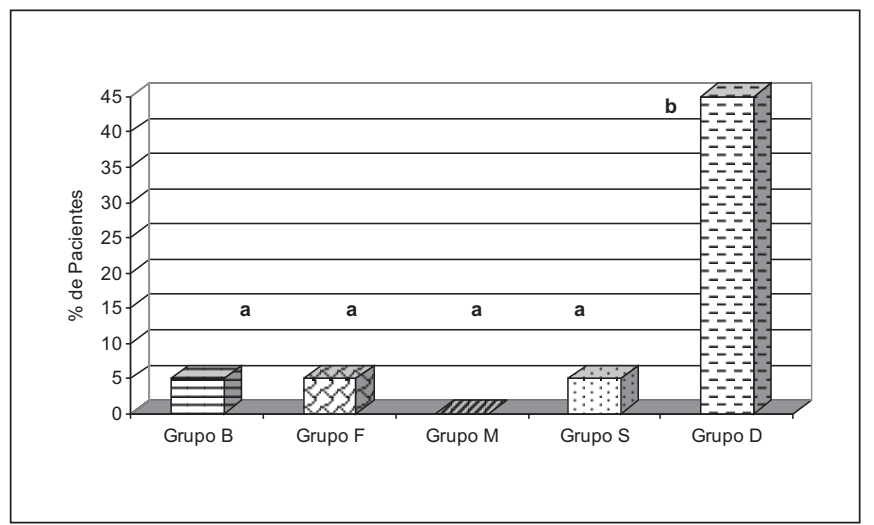

Figura 2 - Necessidade de Analgésicos nas Primeiras 4 Horas (percentagens seguidas de letras são significativamente diferentes pelo teste Qui-quadrado $\mathrm{p}<0,05$ )

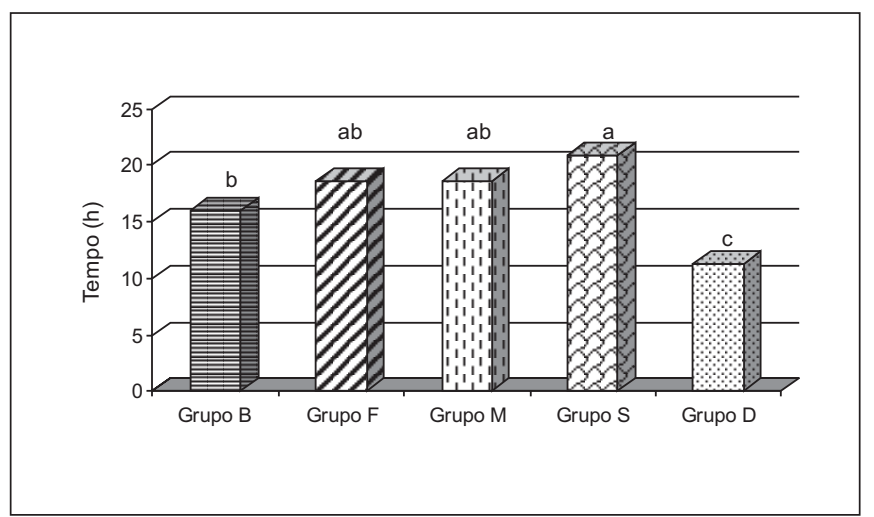

Figura 3 - Tempo Médio de Analgesia nas 24 horas (médias seguidas de letras diferentes são significativamente diferentes pelo teste de Kruskal-Wallis $p<0,05$ )
Efeitos colaterais ocorreram com maior freqüência nos pacientes dos grupos que utilizaram opióides, sendo a incidência mais elevada nos grupos M e S (Figura 4).

Observou-se correlação positiva e significativa $(p<0,05)$ entre os valores obtidos com as duas escalas de avaliação de dor, nos tempos similares, a partir dos 30 minutos até a quarta hora de avaliação (Tabela IV).

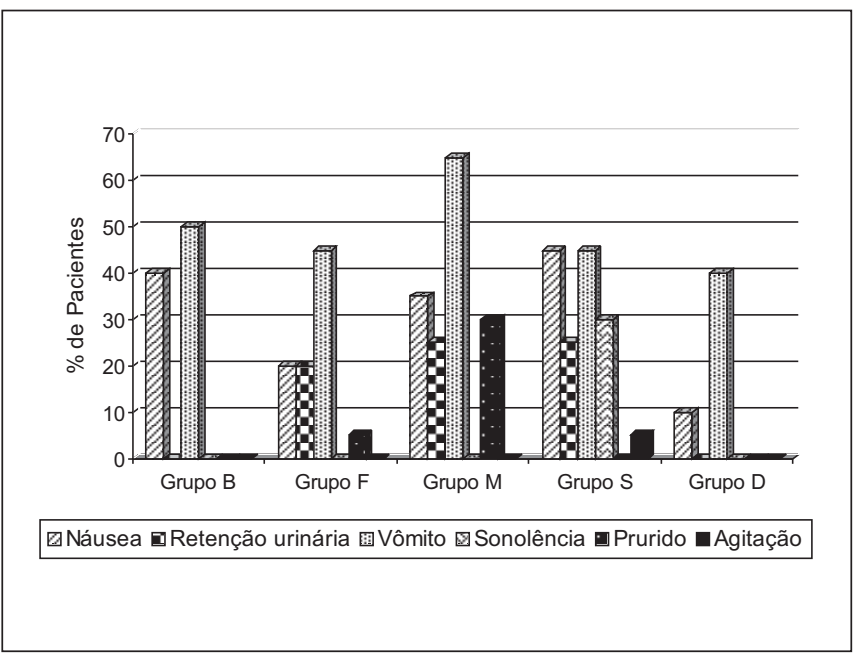

Figura 4 - Incidência dos Efeitos Colaterais nos 5 Grupos

\section{DISCUSSÃO}

Apesar da existência de vários métodos capazes de prevenirem e tratarem segura e efetivamente a dor pós-operatória de pacientes pediátricos, sabe-se que isso não vem ocorrendo e que a dor em crianças ainda é subestimada e negligenciada por equipes médicas e de enfermagem ${ }^{3,11,12}$. As razões que podem explicar esse tratamento inadequado da dor em crianças, quando comparado ao controle da dor pós-operatória em adultos, são inúmeras, sendo mais freqüentemente relacionadas ao receio do aparecimento de efeitos colaterais dos opióides e à crença, bastante difundida, de que crianças possuem reações do tipo decorticação após estímulos nociceptivos, não localizando os estímulos dolorosos, não me-

Tabela IV - Matriz de Coeficiente de Correlação de Pearson entre os Escores das Escalas EADD e EAA

\begin{tabular}{lccccccc}
\hline & EAA 15 & EAA 30 & EAA 45 & EAA 60 & EAA 120 & EAA 180 & EAA 240 \\
\hline EADD 15 & 0,24 & 0,18 & 0,26 & 0,14 & $-0,07$ & $-0,14$ & $-0,06$ \\
EADD 30 & 0,02 & $0,52^{\dagger}$ & 0,29 & $0,34^{*}$ & 0,01 & 0,03 & $-0,06$ \\
EADD 45 & $-0,23$ & $0,36^{*}$ & $0,34^{*}$ & $0,30^{*}$ & 0,01 & 0,00 & $-0,01$ \\
EADD 60 & $-0,08$ & 0,20 & 0,14 & $0,34^{*}$ & 0,16 & 0,06 & 0,01 \\
EADD 120 & 0,28 & 0,20 & 0,21 & 0,25 & $0,49^{\dagger}$ & $0,31^{*}$ & 0,08 \\
EADD 180 & 0,28 & 0,20 & 0,17 & 0,05 & $0,26^{*}$ & $0,35^{*}$ & 0,07 \\
EADD 240 & 0,21 & 0,18 & $-0,01$ & $-0,09$ & 0,20 & 0,20 & $0,63^{\dagger}$ \\
\hline
\end{tabular}

${ }^{*} p<0,05 ;{ }^{\dagger} p<0,01$ 
morizando experiências dolorosas e sendo neurologicamente imaturas para integrarem a percepção nociceptiva no córtex ${ }^{8,13}$

A partir do final da década de 80 , a literatura tem contribuído muito para reverter esse quadro, demonstrando que a experiência dolorosa é uma sensação muito precoce no desenvolvimento do ser humano ${ }^{3,5,14}$ e que o alívio da dor, em qualquer faixa etária, mesmo que eventualmente acompanhado de alguns efeitos colaterais, é o único meio capaz de atenuar os danos provocados pela dor pós-operatória ${ }^{4}$.

As dificuldades na avaliação da dor em crianças foram, em parte, responsáveis por condutas recentes, preconizadas por Ecoffey ${ }^{15}$, sugerindo que técnicas de analgesia sejam administradas antes do despertar da anestesia e sempre que possível evitadas injeções intramusculares ou subcutâneas. A via peridural caudal, em crianças, é muito adequada para administração de agentes analgésicos e vários trabalhos já foram realizados utilizando anestésicos locais, especialmente a bupivacaína, com resultados bastante satisfatórios ${ }^{16-26}$. Procedimentos cirúrgicos mais complexos e com tempo prolongado têm exigido técnicas de analgesia mais efetivas e duradouras, e o uso de opióides, por via peridural caudal, inicialmente com a morfina, tornou-se uma opção para crianças, do mesmo modo que vinha sendo realizado em adultos ${ }^{27-34}$. A falta de uniformidade nas doses de morfina espinhal em crianças, encontrada na literatura, o temor das complicações que poderiam ocorrer com esta técnica e alguns relatos de depressão respiratória tardia em crianças com idade inferior a 12 meses e com doses elevadas retardaram, por muito tempo, a expansão do uso de opióides espinhais na analgesia pediátrica. Autilização de opióides lipossolúveis por via peridural caudal, em dose única, com o objetivo de promover analgesia pós-operatória em crianças tem sido pouco relatada e os resultados não têm sido uniformes. Tempo prolongado de analgesia após administração de fentanil por via peridural caudal, em dose única, em crianças foi observado por alguns autores ${ }^{15,35}$, enquanto outros ${ }^{36}$ não encontraram diferença no tempo de analgesia em relação à bupivacaína pela mesma via. Posteriormente, Benlabed e col. ${ }^{37}$ administraram o sufentanil caudal em dose única em uma série de crianças, para analgesia pós-operatória, promovendo analgesia de instalação rápida e de curta duração.

A utilização de antiinflamatórios não esteróides (AINE) na analgesia de crianças é uma técnica muito difundida, não estando ainda bem estabelecidos a classe de AINE, a dose, a via e o momento de administração que trariam os melhores benefícios para o alívio da dor pós-operatória. Inúmeros trabalhos foram realizados com estas drogas e a maioria deles 38-39 não tem conseguido demonstrar eficácia como analgésico único.
No presente estudo, optou-se pelas doses de opióides, por via peridural caudal, que se revelaram mais seguras e efetivas, em crianças, em trabalhos anteriores, e pelas doses de bupivacaína caudal e diclofenaco retal já consagradas na literatura. O resultado relevante, neste estudo, foi o tempo de analgesia obtido com os opióides lipofílicos, por via caudal, em que o sufentanil caudal promoveu a analgesia mais prolongada e que juntamente com o fentanil não diferiram do tempo de analgesia obtido com a morfina, que é um opióide hidrossolúvel, e portanto com tempo de duração previsível bem mais prolongado. Das hipóteses levantadas para explicar esses resultados, poderíamos citar a ligação mais intensa do fentanil e sufentanil à gordura peridural, abundante em crianças, com liberação lenta e fracionada dos mesmos, por algumas horas, prolongando o tempo de analgesia; o volume de diluição (10 $\mathrm{ml})$, importante no prolongamento do efeito dos opióides lipossolúveis e a dose reduzida de morfina utilizada neste trabalho, partindo da premissa que exista uma relação direta entre a dose de opióide hidrossolúvel e o tempo de analgesia.

O comportamento semelhante dos grupos B, F, M e S nas primeiras 4 horas de analgesia pós-operatória, em que somente $5 \%$ dos pacientes necessitaram de complementação analgésica em relação a $45 \%$ dos pacientes do grupo $D$ que necessitaram analgesia complementar vem ao encontro de outro estudo ${ }^{40}$ que afirma não serem os antiinflamatórios suficientemente potentes para aliviarem a dor pós-operatória imediata.

Para testar a confiabilidade dos testes empregados neste estudo, pesquisou-se a existência de correlação entre os mesmos. Observou-se correlação positiva entre os dois métodos a partir dos 30 minutos, o que reforçou a consistência das avaliações.

O maior número de efeitos adversos, embora de pequena gravidade, incidiu nos pacientes que receberam opióides espinhais, especialmente nos grupos S e M. Náuseas e vômitos não diferiram entre os 5 grupos, em relação à incidência, e foram os únicos efeitos adversos dos grupos $D$ e $B$.

Outros estudos clínicos de analgesia pós-operatória, especialmente com opióides espinhais, serão necessários para determinar qual o agente e a técnica de administração mais seguros e efetivos para serem empregados em pacientes pediátricos; no entanto, no presente estudo, evidenciou-se que doses seguras de morfina peridural caudal promovem analgesia de mesma qualidade, duração e incidência de efeitos colaterais do que injeções únicas de fentanil ou sufentanil pela mesma via; que a bupivacaína caudal promove analgesia prolongada e efetiva, com reduzida incidência de efeitos colaterais e que o diclofenaco por via retal, embora com baixa incidência de efeitos adversos, não proporciona analgesia adequada, mesmo nas primeiras horas do pós-operatório. 


\section{Postoperative Analgesia in Pediatric Patients: Comparative Study among Local Anesthetics, Opioids and Non-Steroidal Anti-Inflammatory Drugs}

Miriam Seligman Menezes, TSA, M.D., Judymara Lauzi Gozzani, TSA, M.D.

\section{INTRODUCTION}

After having been proven that children perceive, respond and remember pain similarly to adults, several analgesic techniques, especially for postoperative pain relief, have been proposed aiming at protecting children against metabolic, hemodynamic and psychological changes caused by surgical procedures ${ }^{1-5}$.

Pain evaluation has been considered a major difficulty in handling postoperative pain in children below 6 years of age, and this has led anesthesiologists to chose techniques that would promote analgesia even before emergence, thus helping this evaluation ${ }^{6-8}$.

Systemic analgesics administered by different routes and loco-regional blockades are common techniques for children's postoperative pain control, but still today, medical and nursing teams are concerned with the pediatric use of opioids due to the risk of side-effects.

This study aimed at evaluating and comparing different techniques of postoperative analgesia in children. Drugs studied were epidural caudal bupivacaine, morphine, fentanyl and sufentanil, and rectal diclofenac administered after anesthesia induction. Quality of analgesia was evaluated in the first 4 hours by two different pain evaluation methods, and evaluation methods reliability, total analgesia duration and side-effects during the first 24 postoperative hours were observed.

\section{METHODS}

After the Ethical Committee approval and written consent of parents or guardians, participated in this study 100 children of both genders, aged 2 to 12 years, physical status ASA I, II and III scheduled for lower limb orthopedic surgeries, urogenital, anal and infraumbilical procedures, who were systematically included, that is, the first two children would belong to Group B, the next two to Group F, and so on, until a total of 20 patients per group.

When needed, patients were premedicated with oral or nasal midazolam $\left(0.5 \mathrm{mg} . \mathrm{kg}^{-1}\right) 20$ or 30 minutes before the procedure. Anesthesia was induced with halothane in increasing concentrations and nitrous oxide:oxygen (1:1), or with intravenous thiopental; the same inhaled agents were used for maintenance through tracheal tube, laryngeal mask or oropharyngeal airway with assisted or controlled ventilation in a system without $\mathrm{CO}_{2}$ reabsorber. Succinylcholine, atracurium or deep inhalation anesthesia were used to help tracheal intubation. Monitoring consisted of cardioscope, pulse oximetry, pre-cordial stethoscope and non-invasive blood pressure.

Soon after anesthetic induction, epidural caudal block was performed in group B with $0.25 \%$ bupivacaine with epinephrine $(1: 400,000) 0.5$ to $1 \mathrm{ml}^{\mathrm{kg}} \mathrm{kg}^{-1}$ according to the incision area; epidural caudal block was performed in group $\mathrm{F}$ with fentanyl $\left(1.5 \mu \mathrm{g} . \mathrm{kg}^{-1}\right)$ in $10 \mathrm{ml}$ saline; group $\mathrm{M}$ patients received epidural caudal morphine $\left(30 \mu \mathrm{g} . \mathrm{kg}^{-1}\right)$ in $10 \mathrm{ml}$ saline; by the same route, Group S patients received sufentanil $(0.3$ $\left.\mu \mathrm{g} . \mathrm{kg}^{-1}\right)$ in $10 \mathrm{ml}$ saline. Group D patients received rectal 1 $\mathrm{mg} \cdot \mathrm{kg}^{-1}$ diclofenac.

All patients received oxygen under mask in the immediate postoperative period and were monitored with pulse oximetry. Two different pain evaluation scales were used during the first 4 postoperative hours: Pain and Discomfort Evaluation Scale (PDES) ${ }^{9}$ shown in table I and Self-Evaluation Scale (SES) ${ }^{10}$, according to figure 1. Evaluations were performed at 15-minute intervals for one hour and then at every 60 minutes for four hours, and oral analgesics were administered whenever children reached scores equal to 7 or above in the Pain and Discomfort Evaluation Scale. From this period on, children were evaluated by the nursing team or by the parents based on changes in children's behavior and/or position and trying to relate these events to the presence of pain and need for analgesic complementation. Oral acetominophen ( 10 to $\left.15 \mathrm{mg} \cdot \mathrm{kg}^{-1}\right)$, intravenous metochlopramide $\left(0.15 \mathrm{mg} \cdot \mathrm{kg}^{-1}\right)$ and/or naloxone ( 0.5 to 4 $\left.\mu \mathrm{g} . \mathrm{kg}^{-1}\right)$ were preconized for pain, nausea and/or vomiting and other opioid-related side-effects.

Table I - Pain and Discomfort Evaluation Score (PDES)

\begin{tabular}{|c|c|c|}
\hline Parameters & Criteria & Score \\
\hline \multirow[t]{3}{*}{ Blood pressure } & Up to $20 \%$ of preoperative values & 0 \\
\hline & $20-30 \%$ of preoperative values & 1 \\
\hline & $>30 \%$ of preoperative values & 2 \\
\hline \multirow[t]{3}{*}{ Weeping } & Without weeping & 0 \\
\hline & Weeping, responding to kindness & 1 \\
\hline & Weeping, not responding to kindness & 2 \\
\hline \multirow[t]{3}{*}{ Movements } & None & 0 \\
\hline & Unquiet & 1 \\
\hline & Agitated & 2 \\
\hline \multirow[t]{3}{*}{ Anxiety } & Sleeping or calm & 0 \\
\hline & Moderate & 1 \\
\hline & Very anxious & 2 \\
\hline \multirow[t]{3}{*}{ Posture } & Without specific posture & 0 \\
\hline & Flexion of legs and thighs & 1 \\
\hline & Holding the operated site & 2 \\
\hline \multirow[t]{3}{*}{ Pain complaint } & No pain & 0 \\
\hline & Non-localized pain & 1 \\
\hline & Localized pain & 2 \\
\hline
\end{tabular}

Revista Brasileira de Anestesiologia Vol. 52, N 2, Março - Abril, 2002 


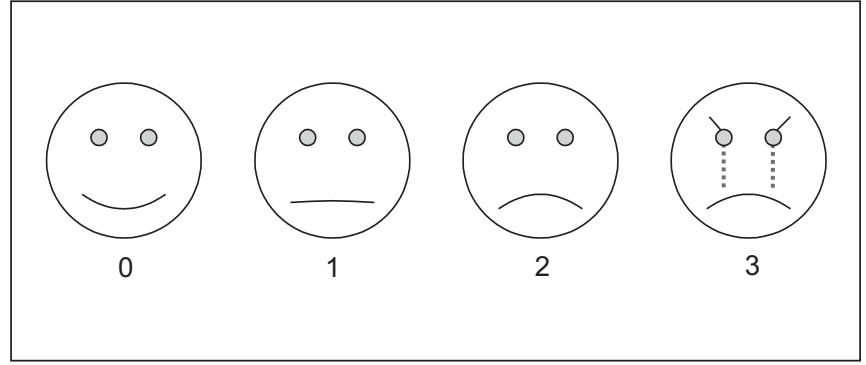

Figure 1 - Self-Evaluation Score (SES)

Statistical analysis was performed by the $\mathrm{F}$ and Chi-square tests to compare demographics among groups; Kruskal-Wallis test was used to compare pain scores and mean analgesia duration among groups; Chi-square test was used to evaluate the need for analgesics during the first 4 hours and the incidence of side-effects in each group;
Pearson correlation study was used to evaluate the correlation between both pain evaluation scales. $P<0.05$ was considered statistically significant.

\section{RESULTS}

There were no significant demographic differences among all groups (Table II).

There were significant differences in mean pain scores among groups in both scales until 60 minutes of evaluation; after this, pain scores did not significantly differ. Group D pain scores were higher as compared to other groups during the first 30, 45 and 60 minutes of evaluation (Table III).

Groups B, F, M and S presented a similar behavior with regard to decreased analgesia needs during the first evaluation hours, significantly differing $(p<0.05)$ from Group $D$ patients who had a very high need for analgesics during the first 4 hours (Figure 2).

Table II - Demographics Data

\begin{tabular}{|c|c|c|c|c|c|}
\hline & Group B & Group F & Group M & Group S & Group D \\
\hline Age (months) * & $\begin{array}{c}67.80 \pm 29.04 \\
(25-120)\end{array}$ & $\begin{array}{c}68.45 \pm 40.60 \\
(24-136)\end{array}$ & $\begin{array}{c}65.45 \pm 31.69 \\
(27-133)\end{array}$ & $\begin{array}{c}80.15 \pm 22.07 \\
48-127)\end{array}$ & $\begin{array}{c}73.40 \pm 36.19 \\
(28-144)\end{array}$ \\
\hline \multicolumn{6}{|l|}{ Gender } \\
\hline Male & 15 & 18 & 14 & 15 & 15 \\
\hline Female & 5 & 2 & 6 & 5 & 5 \\
\hline \multicolumn{6}{|l|}{ ASA } \\
\hline I & 16 & 17 & 16 & 16 & 18 \\
\hline II & 2 & 3 & 2 & 3 & 2 \\
\hline III & 2 & - & 2 & 1 & - \\
\hline
\end{tabular}

*Values in Mean $\pm S D$ and minimum and maximum values in brackets; $p<0.05$ by $F$ and Chi-square tests

Table III - Comparison Among Groups of Pain Scores Determined by Pain and Discomfort Evaluation Score (PDES) and Self-Evaluation Score (SES)

\begin{tabular}{|c|c|c|c|c|c|c|c|c|}
\hline Variables & Group B & Group F & Group M & Group S & Group D & $x$ & $x^{2}$ & $p<$ \\
\hline \multicolumn{9}{|l|}{ PDES } \\
\hline 15 & $0.40 c$ & $1.45 a b$ & $0.75 \mathrm{bc}$ & $0.70 \mathrm{bc}$ & $2.55 a$ & 1.17 & 10.42 & 0.03 \\
\hline 30 & $1.15 c$ & $1.40 \mathrm{~b}$ & $1.05 c$ & $1.20 c$ & $3.35 a$ & 1.63 & 10.88 & 0.02 \\
\hline 45 & $0.55 c$ & $1.00 \mathrm{~b}$ & $1.00 \mathrm{~b}$ & $0.60 \mathrm{c}$ & $3.00 a$ & 1.34 & 14.72 & 0.005 \\
\hline 60 & $0.55 c$ & $1.00 b$ & $0.60 c$ & $0.60 \mathrm{c}$ & $2.45 a$ & 1.04 & 21.71 & 0.0002 \\
\hline 120 & 0.50 & 0.40 & 0.15 & 0.60 & 0.95 & 0.52 & 8.83 & 0.06 \\
\hline 180 & 0.10 & 0.50 & 0.70 & 0.75 & 0.70 & 0.55 & 3.13 & 0.63 \\
\hline 240 & 0.85 & 0.70 & 0.30 & 0.80 & 0.80 & 0.69 & 1.69 & 0.79 \\
\hline \multicolumn{9}{|l|}{ SES } \\
\hline 15 & 1.40 & 1.00 & 1.00 & 1.33 & 0.00 & 1.167 & 2.02 & 0.73 \\
\hline 30 & 1.00 & 1.33 & 1.40 & 1.33 & 1.33 & 1.233 & 0.61 & 0.96 \\
\hline 45 & 0.916 & 1.40 & 0.778 & 1.00 & 1.25 & 1.024 & 1.63 & 0.80 \\
\hline 60 & 0.785 & 1.00 & 1.00 & 0.636 & 1.09 & 0.892 & 1.56 & 0.81 \\
\hline 180 & 0.182 & 0.545 & 0.333 & 0.20 & 0.143 & 0.272 & 4.58 & 0.33 \\
\hline 240 & 0.200 & 0.273 & 0.571 & 0.333 & 0.429 & 0.377 & 1.12 & 0.89 \\
\hline
\end{tabular}

Means followed by different letters are significantly different by Kruskal-Wallis test $(p<0.05)$ 


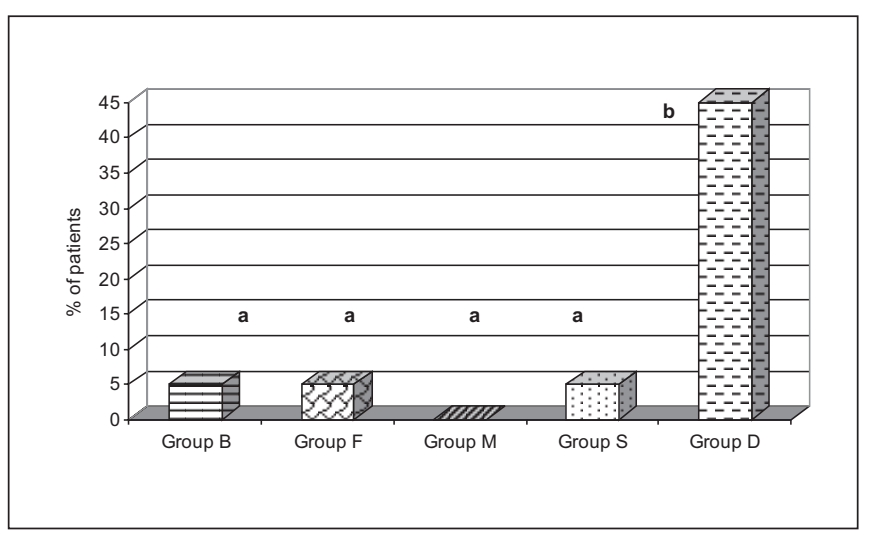

Figure 2 - Analgesic Need During the First 4 Hours (percentages followed by letters are significantly different by Chi-square test $p<0.05)$

Total analgesia duration during 24 hours was decreased in Group D with statistically significant differences as compared to other groups (Figure 3). Analgesia was longer in Group $S$ without significant differences as compared to groups $\mathrm{F}$ and $\mathrm{M}$, but significantly different as compared to Groups $D$ and $B$. There were no significant differences in analgesia duration among groups $\mathrm{M}, \mathrm{F}$ and $\mathrm{B}$.

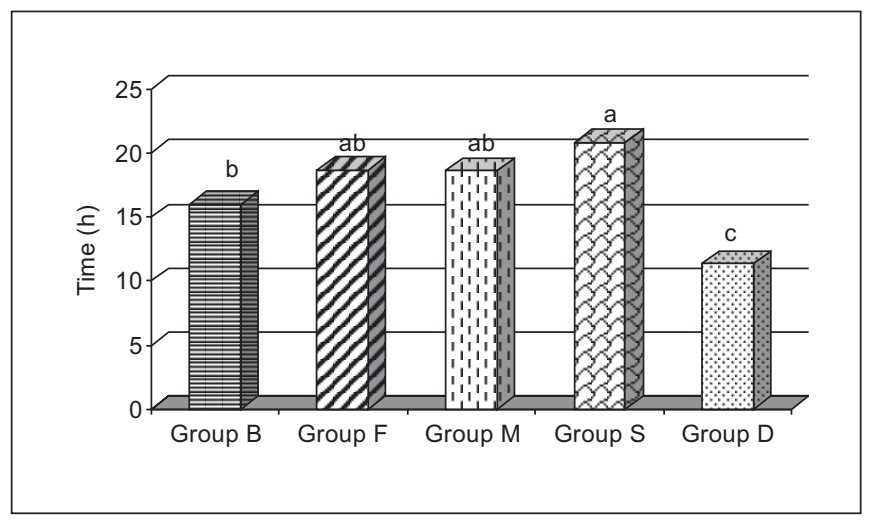

Figure 3 - Mean Analgesia Duration in 24 hours (means followed by different letters are significantly different by Kruskal-Wallis test $p<0.05)$
Side-effects were more frequent in patients using opioids with a higher incidence in groups $\mathrm{M}$ and $\mathrm{S}$ (Figure 4).

There has been a positive and significant correlation ( $p<$ 0.05 ) between both pain evaluation scales in similar moments, as from 30 minutes until the $4^{\text {th }}$ evaluation hour (Table IV).

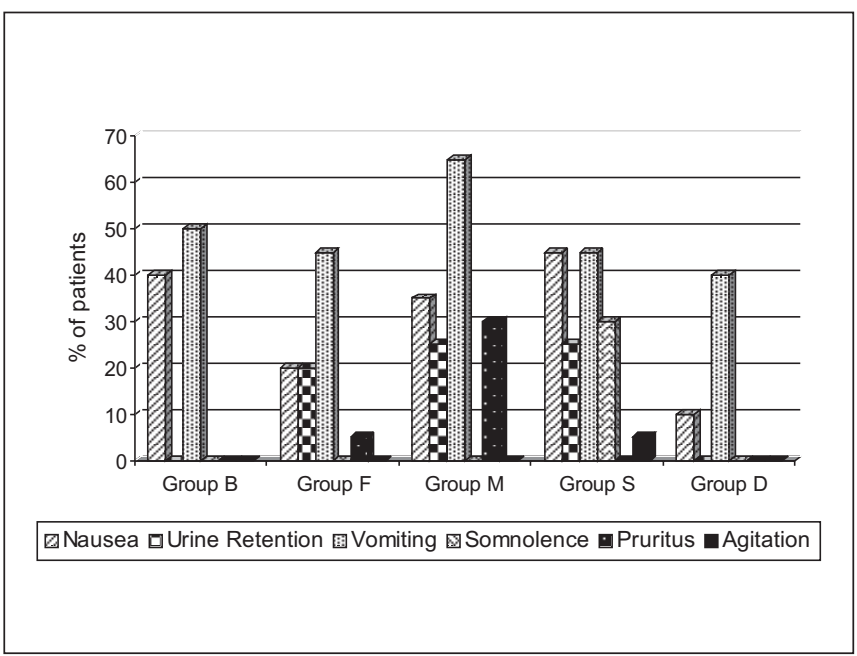

Figure 4 - Side-Effects in All Groups

\section{DISCUSSION}

Although several methods to prevent and safely and effectively treat postoperative pediatric pain, it is known that they are not being used and that pediatric pain is still underestimated and neglected by medical and nursing teams ${ }^{3,11,12}$. There are many reasons to explain this inadequate pain treatment in children, as compared to postoperative pain control in adults, which more often related to fear of opioid-induced side-effects and to the widely spread belief that children have decortication-type reactions after noxious stimuli, not localizing pain stimuli, not memorizing painful experiences and being neurologically immature to integrate noxious perception to the cortex ${ }^{8,13}$.

Table IV - Pearson Correlation Coefficient Matrix Between PDES and SES Scores

\begin{tabular}{lcclllll}
\hline & SES 15 & SES 30 & SES 45 & SES 60 & SES 120 & SES 180 & SES 240 \\
\hline PDES 15 & 0.24 & 0.18 & 0.26 & 0.14 & -0.07 & -0.14 & -0.06 \\
PDES 30 & 0.02 & $0.52^{\dagger}$ & 0.29 & $0.34^{*}$ & 0.01 & 0.03 & -0.06 \\
PDES 45 & -0.23 & $0.36^{*}$ & $0.34^{*}$ & $0.30^{*}$ & 0.01 & 0.00 & -0.01 \\
PDES 60 & -0.08 & 0.20 & 0.14 & $0.34^{*}$ & 0.16 & 0.06 & 0.01 \\
PDES 120 & 0.28 & 0.20 & 0.21 & 0.25 & $0.49^{\dagger}$ & $0.31^{*}$ & 0.08 \\
PDES 180 & 0.28 & 0.20 & 0.17 & 0.05 & $0.26^{*}$ & $0.35^{*}$ & 0.07 \\
PDES 240 & 0.21 & 0.18 & -0.01 & -0.09 & 0.20 & 0.20 & $0.63^{\dagger}$ \\
\hline
\end{tabular}

${ }^{*} p<0.05 ;{ }^{\dagger} p<0.01$ 
As from the late $80 \mathrm{~s}$, literature has contributed to revert this situation showing that painful experiences are a very early sensation in human development ${ }^{3,5,14}$ and that pain relief, in any age-even if followed by some side-effects, is the only means to attenuate postoperative pain damages ${ }^{4}$.

Difficulties in evaluating pediatric pain were partially responsible for recent approaches suggested by Ecoffey ${ }^{15}$, who suggests that analgesia should be administered before emergence and that muscular or subcutaneous injections should be avoided whenever possible. Epidural caudal administration in children is very adequate for analgesics administration and several studies were carried out using local anesthetics, especially bupivacaine, with satisfactory results ${ }^{16-26}$. Complex and prolonged surgical procedures have required more effective and long-lasting analgesic techniques and epidural caudal opioids, initially morphine, have become an option to children, similarly to what has been used to adults ${ }^{27-34}$. The lack of spinal morphine dose uniformity in the literature, the fear of complications which could result from this technique and some late respiratory depression reports in children below 12 months of age under high doses have delayed for a long time the widespread use of spinal opioids for pediatric analgesia. Epidural caudal liposoluble opioids in a single dose aiming at promoting postoperative analgesia in children are few in the literature and results are not uniform. Prolonged analgesia after epidural caudal fentanyl in a single dose in children has been observed by some authors ${ }^{15,35}$, while others ${ }^{36}$ have not seen differences in analgesia duration with bupivacaine by the same route. Benlabed et al. ${ }^{37}$ have administered a single dose of caudal sufentanil in children for postoperative analgesia, and were able to promote fast onset and short duration analgesia. Non-steroid anti-inflammatory drugs (NSAIDs) for pediatric analgesia is a very popular technique but NSAIDs class, dose, route and administration time to promote optimal postoperative pain relief are not yet well established. Several studies were performed with this drug and most of them ${ }^{38-39}$ have not been able to show its efficacy as a single drug. In our study, we decided for epidural caudal opioid doses which have shown to be safer and more effective in children in previous studies, and for caudal bupivacaine and rectal diclofenac which are already recognized by the literature. The relevant result of our study was analgesia duration obtained with caudal lipophylic opioids, where sufentanil has promoted more prolonged analgesia and, together with fentanyl, was not different from morphine, a hydrosoluble opioid, thus with more prolonged estimated duration. From the hypotheses raised to explain these results, one may mention the most intense binding of fentanyl and sufentanil to epidural fat, which is abundant in children, with slow and fractionated release for some hours and prolonging analgesia duration; dilution volume $(10 \mathrm{ml})$, important in prolonging liposoluble opioids effect; and the low morphine dose used in this study, supposing that there is a direct relationship between the hydrosoluble opioid dose and analgesia duration. Similar behavior of groups B, F, M and S during the first 4 postoperative analgesia hours, where only $5 \%$ of patients needed analgesic complementation, as compared to $45 \%$ of group D patients who needed additional analgesia, has confirmed another study ${ }^{40}$ which states that anti-inflammatory drugs are not potent enough to relieve immediate postoperative pain.

To check the reliability of the tests used in this study, the presence of correlation between them has been investigated. There has been a positive correlation between both methods after 30 minutes, what has reinforced evaluations consistency.

Patients receiving spinal opioids had more side-effects, although not severe, especially groups $S$ and $M$. Nausea and vomiting did not differ among the 5 groups and were the only adverse effects in groups D and B.

Other postoperative analgesia studies, especially with spinal opioids, will be needed to determine safer and more effective agents and administration techniques for pediatric patients; however our study has shown that safe epidural caudal morphine doses promote analgesia of the same quality, duration and incidence of side-effects than single fentanyl or sufentanil doses by the same route; that caudal bupivacaine promotes prolonged and effective analgesia with minor incidence of side-effects; and that rectal diclofenac, although with a low incidence of side-effects, does not provide an adequate analgesia even during the first postoperative hours.

\section{REFERÊNCIAS - REFERENCES}

01. Meigner M, Zaouter M, Ricard $\mathrm{PH}$ et al - Analgésie per et postopératoire en chirurgie pédiatrique: expérience d'un an. Chir Pediatr, 1990;31:341-344.

02. Altimier L, Norwood S, Dick MJ et al - Postoperative pain management in preverbal children: the prescription and administration in analgesics with and without caudal analgesia. J Pediatr Nurs, 1994;9:226-232.

03. Berde CB - Pediatric postoperative pain management. Pediatric Clin North Am, 1989;36:921-940.

04. Ecoffey C, Desparmet J - Analgesia na Cirurgia Pediátrica, em: Bonnet F - A Dor no Meio Cirúrgico. Porto Alegre, Artes Médicas, 1993;278-294.

05. Desparmet JF - Acute Pain Syndromes in Children, em: Raj PP Pain Medicine. A Comprehensive Review. St. Louis, Mosby, 1996;530-536.

06. McGrath PA - An assessment of children's pain: a review of behavioral, physiological and direct scaling techniques. Pain, 1987;31:147-176.

07. Rice LJ, Pudimat MA, Hannallah RS - Timing of caudal block placement in relation to surgery does not affect duration of postoperative analgesia in paediatric ambulatory patients. Can J Anaesth, 1990;37:429-431.

08. Lloyd -Thomas AR - Pain management in paediatric patients. $\mathrm{Br}$ J Anaesth, 1990;64:85-104.

09. Goldman A, Lloyd-Thomas AR - Pain management in children. Br Med Bull, 1991;47:676-689.

10. Tesler MD, Savedra MC, Holzemer WL et al - The word-graphic rating scale as a measure of children's and adolescents' pain intensity. Res Nurs Health, 1991;14:361-371.

11. Swaford LI, Allan D - Pain relief in the pediatric patient. Med Clin North Am, 1968;52:131-136.

12. Mather L, Mackie $\mathrm{J}$ - The incidence of postoperative pain in children. Pain, 1983;15:271-282. 
13. Dalens B - Regional anesthesia in children. Anesth Analg, 1989;68:654-672.

14. Choonara IA - Pain relief. Arch Dis Child, 1989;64:1101-1102.

15. Ecoffey C - Analgésie postopératoire chez l'enfant: techniques. Cah Anesthesiol, 1995;43:305-306

16. Soliman MG, Ansara S, Laberge R - Caudal anaesthesia in paediatric patients. Can Anaesth Soc J, 1978;25:226-230.

17. Kay B - Caudal block for postoperative pain relief in children. Anaesthesia, 1974;29:610-614.

18. Lunn JN - Postoperative analgesia after circuncision. A randomized comparison between caudal analgesia and intramuscular morphine in boys. Anaesthesia, 1979;34:85-104.

19. Payne KA, Hedrix MR, Wade WJ et al - Caudal bupivacaine for postoperative analgesia in pediatric lower limb surgery. J Pediatr Surg, 1993;28:155-157.

20. Concha M, Gonzáles J, Vergara R - Analgésie postopératoire pour la chirurgie ambulatoire chez l'enfant: comparaison de deux techniques. Cah Anesthesiol, 1994;42:339-342.

21. Broadman LM, Hannalah RS, Norden JM et al - Kiddie caudals: experience with 1154 consecutive cares without complications. Anesth Analg, 1987;66(Suppl):S18

22. Armitage EN - Caudal block in children. Anaesthesia, 1979; $34: 396$.

23. Wolf AR, Valley RD, Fear DW et al - Bupivacaine for caudal analgesia in infants and children: the optimal effective concentration. Anesthesiology, 1988;69:102-106.

24. Dalens B, Hasnaoui A - Caudal anesthesia in pediatric surgery: success rate and adverse effects in 750 consecutive patients. Anesth Analg, 1989;68:654-672.

25. Warner MA, Kunkel SE, Offord KO et al - The effects of age, epinephrine, and operative site on duration of caudal analgesia in pediatric patients. Anesth Analg, 1987;66:995-998.

26. Holthusen H, Eichwede F, Stevens M et al - Preemptive analgesia: comparison of preoperative with postoperative caudal block on postoperative pain in children. $\mathrm{Br} \mathrm{J}$ Anaesth, 1994;73: 440-442.

27. Jensen $\mathrm{BH}$ - Caudal block for postoperative pain relief in children after genital operations. A comparison between bupivacaine and morphine. Acta Anaesthesiol Scand, 1981;25:373-375.

28. Krane EJ, Tyler DC, Jacobson LE - The dose response of caudal morphine in children. Anesthesiology, 1989;71:48-52

29. Valls JM, Mabrok MM, Teixidor PA et al - Analgesia postoperatoria con morfina por via caudal en cirurgia pediátrica: estudio aleatorio y a doble ciego comparada con bupivacaina. Rev Esp Anestesiol Reanim, 1989;36:88-92.

30. Wolf AR, Hughes D, Wade A et al - Postoperative analgesia after paediatric orchidopexy: evaluation of a bupivacaine - morphine mixture. Br J Anaesth, 1990;64:430-435

31. Wolf AR, Hughes D, Hobbs AJ et al - Combined morphinebupivacaine caudals for reconstrutive penile surgery in children: systemic absorption of morphine and postoperative analgesia. Anaesth Intensive Care, 1991;19:17-21.

32. Valley RD, Bailey AG - Caudal morphine for postoperative analgesia in infants and children: a report of 183 cases. Anesth Analg, 1991;72:120-124.

33. Henneberg SW, Hole P, Hass IM et al - Epidural morphine for postoperative pain relief in children. Acta Anaesthesiol Scand, 1993;37:664-667.

34. Irving GA, Butt AD, Vanderveen B - A comparison of caudal morphine given pre or post surgery for postoperative analgesia in children. Pediatr Anaesth, 1993;3:217-221.
35. Alvarez MAP, Menezes MS, Gozzani JL et al - Analgesia pós-operatória com uso de fentanil por via peridural sacra em cirurgia pediátrica. Estudo não comparativo multicêntrico. Rev Bras Anestesiol, 1988;38(Supl):81.

36. Campbell FA, Yentis SM, Fear DW et al - Analgesic efficacy and safety of a caudal bupivacaine-fentanyl mixture in children. Can J Anaesth, 1992;39:661-664.

37. Benlabed M, Ecoffey C, Levron JC et al - Analgesia and ventilatory response to $\mathrm{CO}_{2}$ following epidural sufentanil in children. Anesthesiology, 1987;67:948-951.

38. Moores MA, Wandless JG, Fell D - Paediatric postoperative analgesia. A comparison of rectal diclofenac with caudal bupivacaine after inguinal herniotomy. Anaesthesia, 1990;45: 156-158.

39. Gadiyar V, Gallagher TM, Phil M et al - The effect of a combination of rectal diclofenac and caudal bupivacaine on postoperative analgesia in children. Anaesthesia, 1995;50:820-822.

40. McGrath PJ, Johnson GG - Pain management in children. Can J Anaesth, 1988;35:107-109.

\section{RESUMEN}

Menezes MS, Gozzani JL - Analgesia Pós-Operatoria en Pacientes Pediátricos: Estudio Comparativo entre Anestésico Local, Opioides y Antiinflamatorio no Esteróide

Justificativa y Objetivos - El tratamiento del dolor pós-operatoria en niños ha merecido atención especial en las últimas décadas. El propósito de este estudio fue analizar la analgesia pós-operatoria de niños en lo que se relaciona a la calidad y a la duración de la analgesia, la confianzabilidad de los métodos de evaluación y la incidencia de efectos colaterales decurrentes de las diferentes técnicas de analgesia utilizadas.

Método - Participaron del estudio 100 niños con edades entre 2 y 12 anos distribuidos en 5 grupos de 20 niños cada, que recibieron, luego después la inducción de la anestesia, los siguientes tratamientos de analgesia: grupo $B$, bupivacaína $0,25 \%$, con vasoconstritor, 0,5 a $1 \mathrm{ml}^{\mathrm{kg}}{ }^{-1}$; grupo $F$, fentanil, 1,5 $\mu \mathrm{g} \cdot \mathrm{kg}^{-1}$; grupo M, morfina, $30 \mu \mathrm{g} \cdot \mathrm{kg}^{-1}$, grupo $\mathrm{S}$, sufentanil, 0,3 $\mu \mathrm{g} \cdot \mathrm{kg}^{-1}$, todos por vía peridural caudal y el grupo $D$, que recibió diclofenaco potásico (1 $\mathrm{mg}_{\mathrm{kg}} \mathrm{k}^{-1}$ ) por vía retal. El dolor fue evaluada por 2 métodos distintos: uno predominantemente comportamental, objetivo y el otro de auto-evaluación, subjetivo, durante las primeras 4 horas y a partir de este momento hasta la $24^{a}$ hora. Fueron observados efectos colaterales y fueron tratados.

Resultados - En las primeras 4 horas los pacientes de los grupos B, F, M y S presentaron comportamientos semejantes, con mínimas necesidades de analgesia complementar. En las 20 horas restantes el mayor tiempo de analgesia fue observado en el grupo $S$, no divergiendo del grupo $F$ y $M$, más siendo significativamente superior al tiempo de los grupos $B$ y $D$. Diclofenaco rectal no promovió alivio efectivo del dolor. Mayor incidencia de efectos colaterales ocurrió en el grupo M que no divergió del grupo $S$, más fue significativamente superior a los grupos $F$, B y D. Hubo correlación positiva y significativa entre los resultados de las 2 escalas de evaluación del dolor.

Conclusiones - Los opioides espinales se mostraran seguros y efectivos en la analgesia pós-operatoria en niños, más cuando comparados a la bupivacaína no presentaron diferencias relevantes y presentaron mayor incidencia de efectos colaterales. El diclofenaco por vía retal no se mostró efectivo como analgésico único cuando comparado a las otras técnicas. 